\title{
The Potential of the Water Hammer in Pico-Scale Tidal Power Systems: an Experimental Investigation
}

\author{
A. Roberts ${ }^{*}$, B. Thomas, P. Sewell, N. Aslani \\ Department of Design and Engineering \\ Bournemouth University \\ Poole, United Kingdom \\ *robertsa@bournemouth.ac.uk
}

\author{
S. Balmain, I. Balmain, J. Gillman \\ The Balmain Environmental Trust \\ Shaftesbury, United Kingdom
}

\begin{abstract}
The tidal energy within the seas surrounding the United Kingdom offers a vast renewable resource that is perfectly predictable over long timescales. Currently, the bulk of tidal energy research is focused on developing large devices for the most resource rich locations, which can be many miles from populated areas. The potential of small-scale tidal power from sub-optimal shallow water sites, close to populated regions, has so far been overlooked. Such generation would benefit from reduced transmission losses and contribute towards a distributed electricity grid, helping to overcome the variability of other renewables.
\end{abstract}

This work presents a novel method for generating hydropower using the water hammer effect: a pressure surge that can occur in a pipeline following the abrupt closure of a valve. These pressure surges are used to produce vertical oscillations from horizontally flowing water, allowing power to be generated in a manner analogous to a wave energy convertor. A non-optimised scale model was found experimentally to have a peak available power density of $1.08 \pm 0.25 \mathrm{~kW} / \mathrm{m}^{2}$ and a mean of $0.07 \pm 0.02 \mathrm{~kW} / \mathrm{m}^{2}$. In comparison, the MCT SeaGen S (arguably the most well-developed tidal energy device) is capable of generating $3.18 \mathrm{~kW} / \mathrm{m}^{2}$. With further development, a water hammer device may therefore be useful for generating pico-scale tidal power in slow, shallow water flows.

Keywords-pico-scale hydropower; tidal energy; wave energy conversion; sustainability; water hammer; experimental studies

\section{INTRODUCTION}

There is an estimated $32 \mathrm{GW}$ of tidal stream power available in the waters surrounding the United Kingdom [1]. To date, most of the research and development work into harnessing this resource appears to have focussed on developing large devices to maximise power generation from the most resource-rich locations. Commercial tidal stream devices generally target flow speeds of $2 \mathrm{~m} / \mathrm{s}$ plus, and are typically as large as possible to maximise power generation [2]. For example, the MCT SeaGen device, arguably the most fully-developed tidal stream turbine, is considered viable in depths of $20-40 \mathrm{~m}$ where the peak spring tidal current velocity is greater than $2.25 \mathrm{~m} / \mathrm{s}$ [3]. Meanwhile the MeyGen project aims to deploy six demonstration turbines in the Inner Sound in Pentland Firth, northeast Scotland [4], which is many miles from areas of peak demand.
Thus far, relatively little consideration has been given to the potential of generating small-scale tidal power from less resource rich sites that are close to populated areas. Such generation may be an appealing option for coastal communities as part of a distributed generation strategy, helping to overcome the variability of other renewables and fitting in to a wider context of improvements in sustainability and efficiency.

This work presents an initial experimental investigation into a novel system that may be capable of producing such small-scale power. The system makes use of the water hammer effect: a pressure surge that occurs when a fluid is subjected to a sudden change in momentum [5]. The water hammer is a common occurrence during valve operations in pipelines, where it can cause problems ranging from noise and vibration to catastrophic failure in extreme cases [6]. It may also be used beneficially, however, such as in hydraulic rams. These are $18^{\text {th }}$ century water pumps that are still in use today thanks to their reliability and lack of external power requirements [7].

The speed $(c)$ at which a water hammer pressure surge propagates through a pipe is dependent upon the diameter $(D)$, wall thickness $(e)$ and Young's modulus $(E)$ of the pipe, as well as the pipe support factor $(C)$, and the density $(\rho)$ and bulk modulus of elasticity $(K)$ of the fluid [8]:

$$
c=\sqrt{\frac{1}{\rho\left(\frac{1}{K}+\frac{D}{e E} C\right)}}
$$

For a pipe of length $(L)$ supplied by a reservoir of water, the excess pressure will be reflected once it reaches the reservoir and propagate back towards where it was generated (e.g. a valve) [9]. This will take a period of time $(\tau)$ :

$$
\tau=\frac{2 L}{c}
$$

If the valve closure time $(T)$ is significantly long (such that $T \gg \tau)$, the effects of compressibility can be ignored. For an incompressible fluid undergoing deceleration $(\mathrm{d} v / \mathrm{d} t)$, this will result in a pressure surge $(\Delta p)$ :

$$
\Delta p=\rho L \frac{\mathrm{d} v}{\mathrm{~d} t}
$$




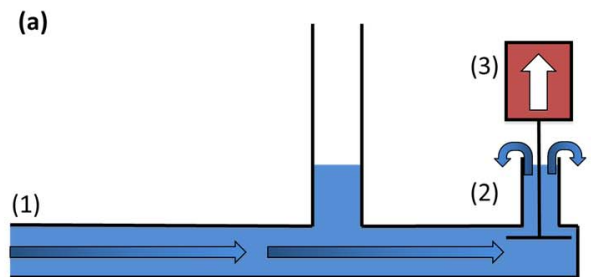

(1) Water enters system at inlet.

(2) Water flows down drive pipe and through check valve at downstream end.

(3) Force of water acting on check valve

causes it to start closing. (b)

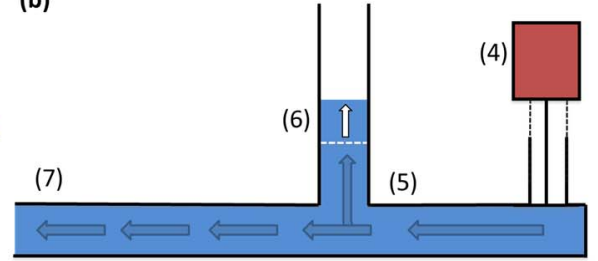

(4) Valve closes, creating water hammer.

(5) Excess pressure propagates from the valve and back up drive pipe.

(6) Water level in air chamber rises due to excess pressure.

(7) System pressure begins to reduce following peak overpressure. (c)

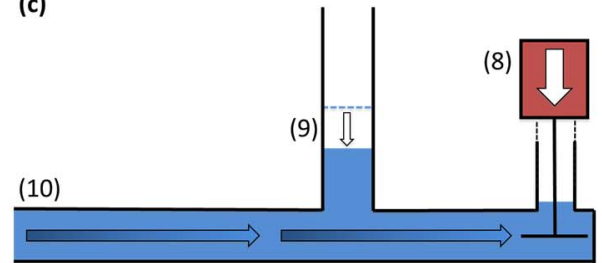

(8) Valve reopens as pressure reduces.

(9) Water level in chamber falls from maximum.

(10) Flow through drive pipe recovers as valve reopens, allowing process to restart.

Fig. 1. Overview of system operation.

Fig. 1 illustrates how these surges can be used to produce a continuously oscillating water level in a vertical chamber from a horizontal flow. This enables power to be generated in a manner analogous to a wave energy convertor (WEC), rather than using traditional hydrokinetic turbines. This appears to be a completely novel method, and may be advantageous in shallow waters where the maximum size of a turbine is constrained by depth. Additionally, from (3), the magnitude of a pressure surge generated by a valve closure is dependent upon the deceleration experienced by the water, rather than its initial velocity. A water hammer driven system may therefore be of use for generating pico-scale hydrokinetic power from sites with low flow-speeds; this work presents an initial experimental investigation into its potential.

\section{Methodology}

To investigate the power available from such a system, a small-scale test rig (illustrated in Fig. 2) was built to conduct physical experiments. The test rig was constructed using threaded sections of $20 \mathrm{~mm}$ diameter PVC pipe (with a bore of $16 \mathrm{~mm}$ ), and a threaded $3 / 4$ inch brass swing-check valve positioned at the downstream end. A hose barb was attached behind this to direct the waste water into a bucket, which served as a sump. The input flow was provided by an 841 reservoir of water located $345 \mathrm{~mm}$ above the drive pipe, with the two connected via $20 \mathrm{~mm}$ diameter PVC hose pipe. A ball valve positioned between the reservoir and the system to control the input and pressure head within the system.

The vertical chamber was made using a $1 \mathrm{~m}$ length of clear, $20 \mathrm{~mm}$ diameter PVC pipe (again with a $16 \mathrm{~mm}$ bore). A 13 $\mathrm{mm}$ diameter cylindrical wooden float, painted bright red, was positioned inside the chamber. The colour of the float contrasted vividly with a white back board positioned behind the test rig, so that motion-tracking techniques could be used to measure the position of the float as a function of time. This was achieved by filming the experimental runs with a 720p HD digital camera operating at 120 FPS, and subsequently processing the recorded videos using a computer code written in MATLAB [10]. This code was created at Bournemouth University, and has previously been used successfully for gait analysis measurements [11]. A calibration video was recorded prior to the experimental runs to account for the angle and position of the camera relative to the float and chamber. Following calibration, the maximum uncertainty in the measurements was found to be of $\pm 0.2 \mathrm{~mm}$ in the central region of the video (corresponding to where the float was located), growing to $\pm 0.8 \mathrm{~mm}$ at the video edges.

The primary aim of the experiments was to identify the power available from the float and to investigate how it is affected by varying the pressure within the system, along with the mass of the float itself. To accomplish this, the motion of 3 different wooden floats (with masses of $4.5 \times 10^{-3}, 8.9 \times$ $10^{-3}$ and $1.39 \times 10^{-2} \mathrm{~kg}$, respectively) was recorded. 10 test runs were conducted per float, with the angle of the ball valve altered for each test run to vary the pressure within the system. In this way, 30 test runs were conducted over the course of the experiments, with each run last approximately $30 \mathrm{~s}$.

Following testing, the video files were processed in MATLAB to extract the raw data for the position $(y)$ of the float against time $(t)$. The data sets for each run were then subdivided into $8.3 \mathrm{~s}$ windows (corresponding to 1000 data points at $120 \mathrm{FPS}$ ). This was done to minimise the variation in the input head due to the diminishing water level within the reservoir, while still providing a meaningful amount of position data for analysis. From the position data, the velocity $(\dot{y})$ and acceleration $(\ddot{y})$ of the float were derived using numerical differentiation. Since that the mass $(m)$ of the float was already known, the total vertical force $\left(F_{t}\right)$ and power $(P)$ of the float could then be calculated according to (4) and (5), respectively.

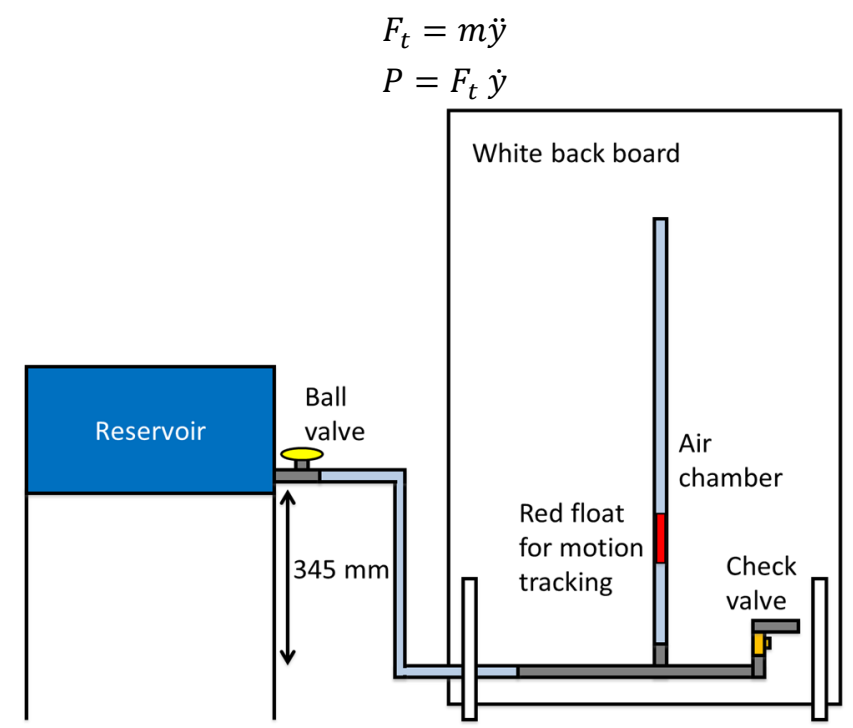

Fig. 2. Experimental set-up. 


\section{RESULTS}

\section{A. Float position, velocity and acceleration}

Fig. 3a illustrates a $2 \mathrm{~s}$ sample period of measured position data as a function of time for a $8.9 \times 10^{-3} \mathrm{~kg}$ float. The shape of the oscillations experienced by the float superficially resembles that of a $|\sin (x)|$ curve; featuring broad, rounded peaks that contrast vividly with the brief, sharp troughs. The troughs correspond to the times at which the check valve closed and generated a pressure surge, causing the water level in the chamber to reverse direction and hence the trajectory of the float to change with it.

In this case, the mean oscillation amplitude was $0.0422 \pm$ $0.001 \mathrm{~m}$, and the mean frequency $3.288 \pm 0.004 \mathrm{~Hz}$. Since the pressure surges driving these oscillations are generated by the closures of the check valve, their frequency is dependent upon how often the check valve closes. Meanwhile, the oscillation amplitude is dependent upon the magnitude of the pressure surges generated. From (3), this is governed by the change in momentum experienced by the water in the pipeline, meaning the amplitude will be dependent upon the speed at which the check valve closes and how long it remains shut for.

In the current system, this behaviour is determined by the forces acting upon the valve, including the drag forces due to the flow, the pressure within the system and the inertia of the valve itself. Nevertheless, it should be possible to use a control system to govern the behaviour of the valve for a given input, and hence regulate the generated oscillations to a desirable range of amplitudes and frequencies for power generation. This could be achieved through the use of pneumatic or solenoid valves, or mechanically using camshafts to couple the valve to the Power Take-Off (PTO).

Another subject of interest in Fig. $3 \mathrm{a}$ is the slight asymmetry in the shape of the float oscillations. In this case, the mean upstroke period was $0.156 \pm 0.005 \mathrm{~s}$, while the mean downstroke period was $0.173 \pm 0.005 \mathrm{~s}$, corresponding to a $0.016 \pm 0.007 \mathrm{~s}$ difference. Although only a slight variation, this is indicative of the difference in the forces acting on the float between the upstroke and downstroke. Due to the inertia of the float, more of its volume will be submerged on the upstroke, meaning it will be propelled upwards by a combination of buoyancy and gradually diminishing excess pressure working against gravity. On the downstroke, however, more of the float is out of the water (again due to inertia), and it is effectively in free-fall.

Fig. $3 \mathrm{~b}$ provides the corresponding velocity-time curve of the data presented in Fig. 3a. Despite the relatively small range of motion, the float reached a peak upwards speed of up to 0.6 $\mathrm{m} / \mathrm{s}$. This maximum occurs shortly after the float has reversed direction, and is sustained only very briefly before gravitational acceleration begins to reduce the speed of both the float and the water in the chamber. Eventually this causes the float to reverse direction, where it reached a peak speed of just over $0.4 \mathrm{~m} / \mathrm{s}$, shortly before the valve closed again and the float was kicked back upwards. This difference is again ascribed to the noninstantaneous nature of the exciting force acting on the float.

Fig. 3c presents the corresponding acceleration-time curve for this data set. The peak acceleration on the float, which corresponds to the pressure surge forcing it upwards, was found to be up to $50 \mathrm{~m} / \mathrm{s}^{2}$. Again, this maximum is sustained only for a very brief period of time, as the acceleration soon falls rapidly to values between 0 and $-10 \mathrm{~m} / \mathrm{s}^{2}$, where it seemingly continues to fluctuate until the next pressure surge is generated at the valve. These fluctuations are ascribed to two factors. Firstly, as described in Section I, the pressure wave generated by a valve closure is reflected back down the drive pipe once it reaches the reservoir. The magnitude of these reflected waves will have been significantly reduced by a

(a) Float position vs. time

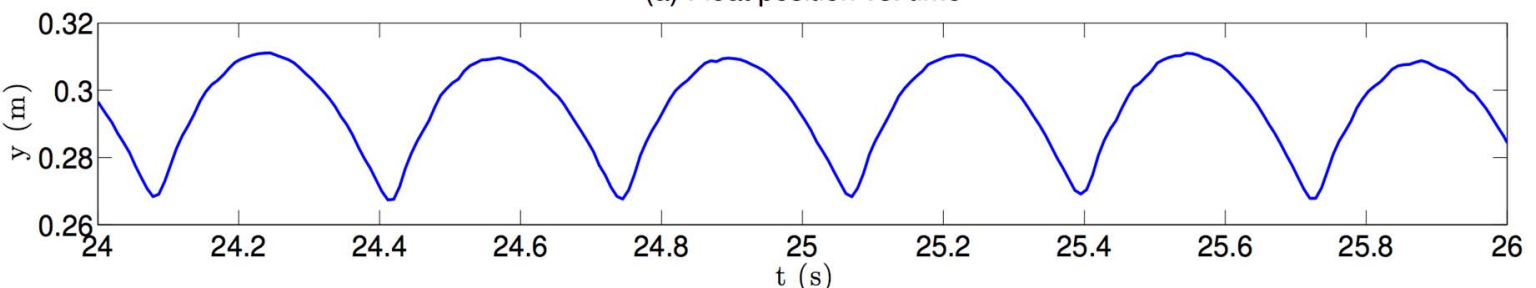

(b) Float velocity vs. time

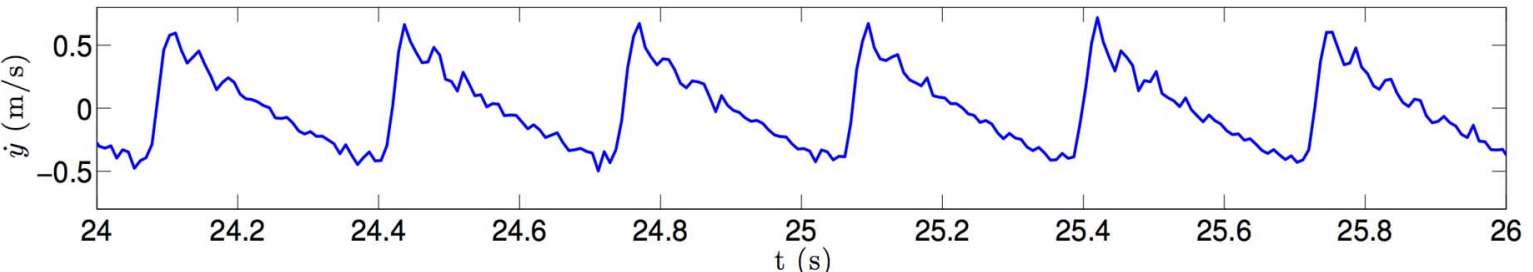

(c) Float acceleration vs. time

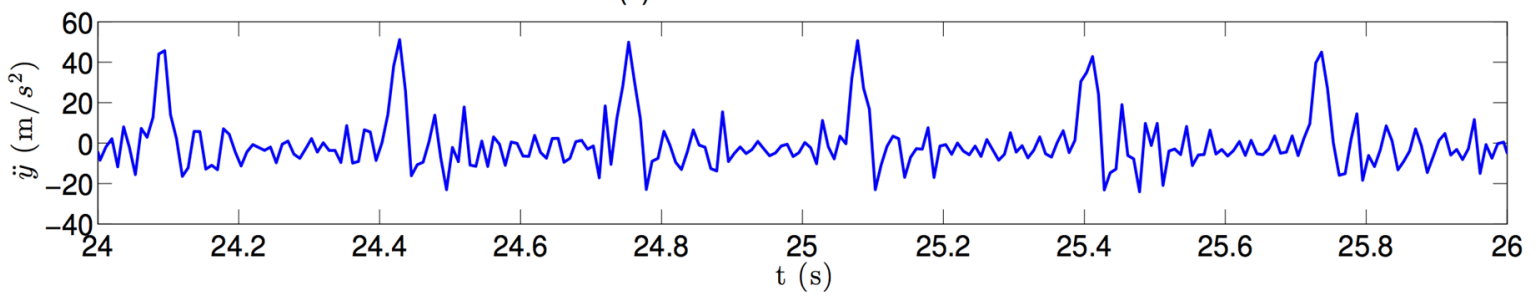

Fig. 3. Sample position, velocity and acceleration curves for the $\mathbf{8 . 9} \times \mathbf{1 0}^{-\mathbf{3}} \mathrm{kg}$ mass float. 


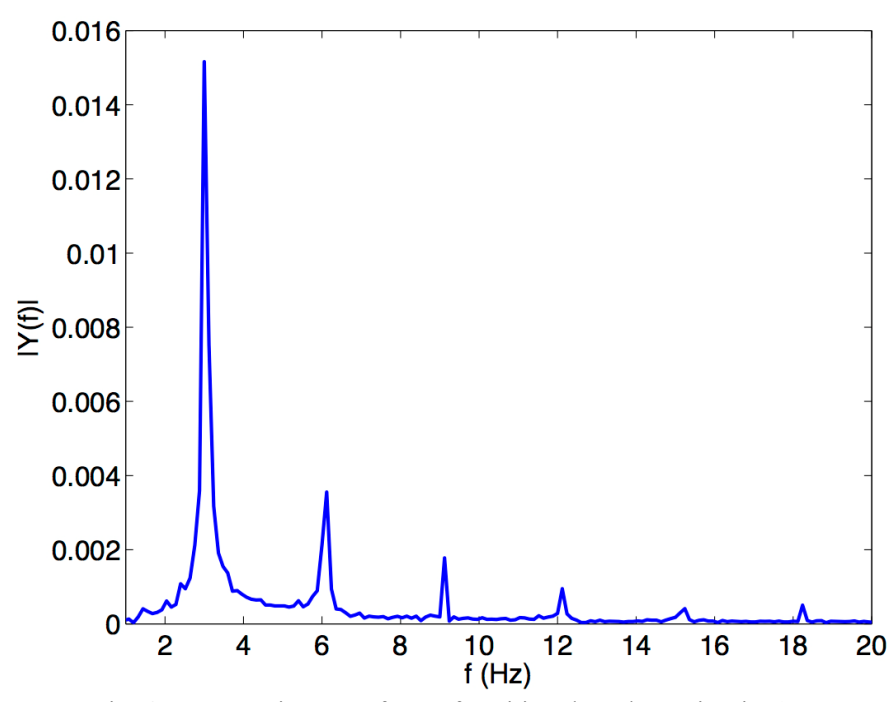

Fig. 4. Fast Fourier Transform of position data shown in Fig. 3a.

combination of friction from the pipe walls and the reflection process itself, meaning their impact on the motion of the float will be significantly smaller than when they first passed underneath it. The components of these waves can be seen in the Fast Fourier Transform (FFT) presented in Fig. 4. The second factor that contributes to these fluctuations is unfortunately noise; a drawback of numerical differentiation is that it serves to amplify any noise present in the original signal [12].

\section{B. Float power}

The power-time curve of the sample data shown in Fig. 3, computed using (4) and (5), is presented in Fig. 5. Even from this brief sample, it can be seen that the power of the float is produced in sharp peaks, which correspond to the point at which the float is forced to reverse direction by a pressure surge. Once the pressure surge has passed, which happens very quickly due its high speed, the only forces acting upon the float are buoyancy and weight; consequently, the power of the float in this region is significantly smaller, particularly considering the low mass of the float $\left(8.9 \times 10^{-3} \mathrm{~kg}\right)$.

To compensate for this, it may be desirable to cap the top of the chamber to trap some air within. As the water level within

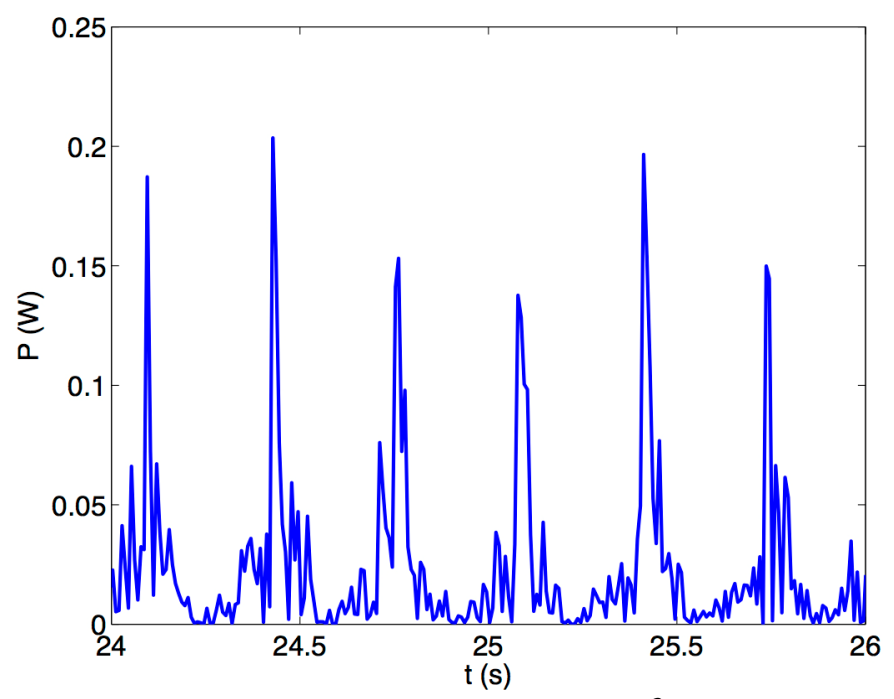

Fig. 5. Sample power-time curve for the $\mathbf{8 . 9} \times \mathbf{1 0}^{-3} \mathrm{~kg}$ mass float. the chamber rises, the air would be compressed, before springing back and contributing to forcing the water level downwards. Although this would reduce the amplitude of the upstroke, it would increase the force on the float on the downstroke, and hence provide a more consistent power output. Capping the chamber would likely affect system behaviour in other ways, however. Since some of the excess pressure would be prevented from escaping out of the top of the chamber, it would likely increase the time required for the pressure within the system to drop sufficiently for the valve to reopen following closure. This would naturally result in less frequent oscillations. Depending upon how long the valve remains closed for, capping the chamber could potentially also alter the form of the float position-time curve, which would have implications for float velocity, acceleration and, therefore, power. This is a subject that will be investigated in future work.

Another noteworthy feature of Fig. 5 is that the power of the float varies from peak to peak, with a maximum of $0.204 \mathrm{~W}$ and a minimum of $0.137 \mathrm{~W}$ visible in Fig. 5. This is ascribed to small variations in the magnitude of the pressure surges generated from one valve closure to the next. Since the valve used to generate the oscillations in the scale model is flowdriven, any variations in the flow will impact upon its closure speed, and hence the magnitude of the pressure surges generated.

Additionally, the peak values that can be seen in Fig. 6 are an order of magnitude greater than the time-average power available to the float, which in this case was $0.018 \mathrm{~W}$. Although these figures are very low, the small cross-sectional area of the scale model $\left(2 \times 10^{-4} \mathrm{~m}^{2}\right)$ limits the size of the float that can be used before frictional effects from the chamber walls become problematic. A larger system would permit more massive floats, which from (5) should increase the power available, assuming the generated pressure surges are capable of overcoming the float inertia.

This can be seen in Fig. 6a and b, which present the mean overall power $(\bar{P})$ and mean peak power $\left(\overline{P_{\text {max }}}\right)$ available from the 3 floats used in the scale model, over a range of mean measured pressure heads within the system $(\bar{\varphi})$. The pressure head was calculated by using a peak analysis method in MATLAB [13] to identify the peaks and troughs within the data. This allowed the mean minimum float position over the course of a test run to be calculated, which was assumed to be indicative of the pressure within the system.

Despite their greater resistance to motion, the heavier floats clearly have more power available to them than the lighter ones, both in terms of peak power and overall power. The variation between the measured data points and the trend is also noticeably greater for the $13.9 \times 10^{-3} \mathrm{~kg}$ float than it is for the lighter floats, particularly in Fig. $6 \mathrm{~b}$. This is because this float was also the longest, and its behaviour was therefore more affected by friction with the chamber walls.

Although it cannot be seen in Fig. 6, there is going to be maximum to this trend of increasing power with increasing mass. As the mass of the float increases, more and more of the pressure surges generated by a given system will go into overcoming its inertia. Consequently, the acceleration, velocity and amplitude of the float will decrease with increasing float 
(a)

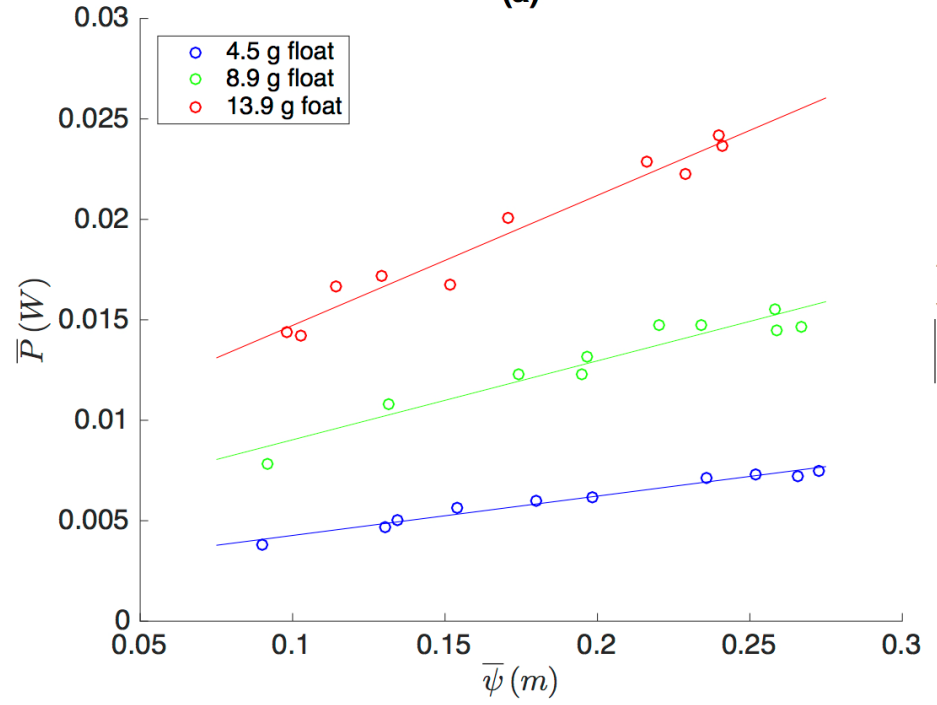

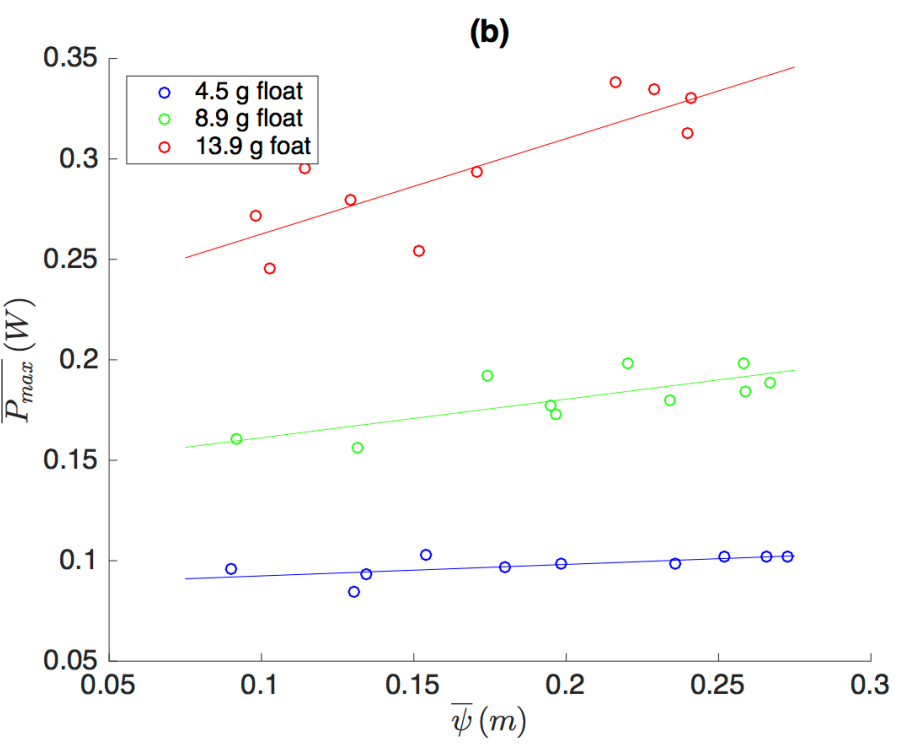

Fig. 6. Effect of mass and system pressure on mean overall (a) and mean peak (b) float power.

mass. Eventually, this decrease in acceleration and velocity will become sufficient to negate the benefit of increasing float mass, and the power available to the float will be seen to start decreasing.

Also of interest in Fig. 6 is the apparent increase in both the mean overall power and the mean peak power with increasing system pressure. This rate of this increase is proportional to float mass; the trend in the mean power of the $13.9 \times 10^{-3} \mathrm{~kg}$ float is noticeably larger than it is for $4.5 \times 10^{-3} \mathrm{~kg}$ float. Calculating the gradient of the trend lines presented in Fig. 6 with this consideration leads to a simple linear relationship for predicting the mean power of the float, as a function of float mass and the pressure head within the system:

$$
\bar{P}=m\left(\beta_{1} \bar{\varphi}+\beta_{2}\right)
$$

Where $\beta_{1}=4.48 \pm 0.16 \mathrm{~m} / \mathrm{s}^{2}$ and $\beta_{2}=0.12 \pm 0.01$ $\mathrm{m}^{2} / \mathrm{s}^{2}$. These constants are likely dependent upon the design of the system, since the power of the float will depend upon the behaviour of the check valve and how much energy is captured from the generated pressure surges. Future work will aim to investigate and quantify the effect of these variables by investigating the effect of different input flow rates, different types of valve and different scale systems.

\section{DisCUSSION}

The results of the experiment presented in this work show that it is possible to produce an oscillating water level in a vertical chamber from a horizontal flow. This is achieved through the use of pressure surges that are created by a repeatedly closing valve. Although the shape of the generated oscillations is different to those of surface gravity waves, this will allow the generation of hydrokinetic power using methods similar to those utilised by WECs, rather than with traditional hydrokinetic turbine systems. For example, bi-directional turbines could be positioned within the chamber, with the aim of creating an oscillating water column style device [14].

The method used in this experiment was similar to a heaving buoy WEC [15], and involved using the oscillations to drive a float up and down. There are several ways in which power could be extracted from such a float. For example, magnets could be attached to it and a coil of wire wrapped around the chamber, creating a linear alternator for direct electricity generation. Alternatively, the float could be connected to a crankshaft to provide rotary mechanical power. This would allow several devices to be coupled to a single crankshaft, which would help to provide a more consistent power output than that seen in Fig. 5, particularly if the crankshaft was connected to a valve control system. Capping the chamber and trapping air within to act as a spring may also help to provide a more consistent power output.

There may be advantages to using such a system, depending upon the characteristics of a given hydropower site. Converting some of the energy of horizontally flowing water into vertical oscillations would eliminate the constraint of water depth on the maximum size of the PTO that could be used. Consequently, a water hammer device may be able to access shallow waters where conventional turbine systems are not considered feasible. This may be particularly appropriate for tidal stream power, where many resources assessments currently neglect waters that are less than $5 \mathrm{~m}$ deep due to technological restrictions (e.g. [1]).

A second potential advantage is that, from (1), the magnitude of a pressure surge generated by a slow valve closure is dependent upon the deceleration experienced by the flow within the pipe, rather than its initial velocity. Assuming the magnitude of this deceleration remains the same, a water hammer device may therefore be capable of functioning just as well in lower speed flows as it does in higher speeds. Although (7) shows that the power of the float in the scale model increased proportionally with pressure, which corresponds to flow speed, this will not be the case for all systems. This is because, in the scale model, the valve generating the pressure surges was driven by the flow through it. As a result, its closure speed varied with input flow, meaning the change in momentum experienced by the water was not consistent. If a controlled valve was used and set such that the water 
experienced the same deceleration regardless of flow speed, it is hypothesised that the mean power would remain constant.

Both of these potential advantages will be dependent upon the power a water hammer driven device can generate, however. The maximum mean peak float power computed from the results of this experiment was $0.34 \pm 0.08 \mathrm{~W}$, which occurred for the heaviest float oscillating in the highest system pressure. The corresponding mean overall power value was computed as $0.023 \pm 0.06 \mathrm{~W}$. Although these figures are extremely small, the very small scale of the test-rig limited the maximum mass of the float that could be used. Normalising the power output for the cross-sectional area of the pipe provides a mean peak power density of $1.08 \pm 0.25 \mathrm{~kW} / \mathrm{m}^{2}$, with a corresponding mean overall power density of $0.07 \pm 0.02$ $\mathrm{kW} / \mathrm{m}^{2}$. Comparatively, the MCT SeaGen S device can generate $3.18 \mathrm{~kW} / \mathrm{m}^{2}$. Although this is quite a crude comparison, given that it does not account for effects of scale (e.g. Reynolds number) or the heavier floats a larger device could accommodate, it suggests that, with further research and development work, a water hammer driven device may have potential for generating pico-scale hydropower. Although the this work is framed within tidal power, a water hammer system would be capable of functioning in a variety of other smallscale hydropower sites, for example shallow rivers and streams, or potentially even in surge tanks in pipe systems.

\section{CONCLUSIONS}

This work has presented an initial small-scale experiment into a novel renewable energy system that creates vertical oscillations from horizontally flowing water. This is achieved through the use of a repeatedly closing valve to create pressure surges. Power can then be generated from these oscillations using a variety of techniques, many of which are already used by WECs. The shape of the generated oscillations is different to those of surface gravity waves, however, meaning a water hammer PTO would need to be optimised differently to those used in ocean wave systems.

The maximum mean peak power of a $13.9 \times 10^{-3} \mathrm{~kg}$ float was computed to be $0.34 \pm 0.08 \mathrm{~W}$, with a corresponding 0.023 $\pm 0.06 \mathrm{~W}$ available over the course of that test run on average. These figures were calculated from the position-time data of the float, which was measured using a motion tracking technique. The power of the float was found to increase with float mass and system pressure, although it is hypothesised that there will be an upper limit to this behaviour for any given system. Additionally, it is predicted that a different system using a controlled valve, rather than a flow driven one, would be capable of producing power independent of the flow speed, if desired. This means a water hammer system may be suited for power generation from low speed sites, assuming an efficient device can be designed.

Another potential advantage of a water hammer system lies in shallow water applications. Since energy is extracted from vertical oscillations rather than the flow itself, the maximum size of the PTO would not be constrained by water depth. This may be particularly advantageous in tidal stream applications, where sites that are less than $5 \mathrm{~m}$ deep are currently neglected from resource assessments due to technological limitations.

Both of these advantages will depend upon whether an efficient device can be designed and the power it can generate, however. Future work will aim to further quantify the effects of float mass, device size, input flow rate and capping the chamber on performance. Additional work will focus on building a mechanical PTO and coupling several devices onto a single crankshaft, to further investigate power output. Depending upon the results of these investigations, a water hammer system may be a useful device for generating picoscale tidal power in slow, shallow water flows.

\section{ACKNOWLEDGMENTS}

The authors would like to acknowledge and thank Bournemouth University and the Balmain Environmental Trust for funding this research.

\section{REFERENCES}

1. The Crown Estate. UK Wave and Tidal Key Resource Areas Project: Summary Report. 2012; Available from: http://www.thecrownestate.co.uk/media/5476/uk-wave-and-tidalkey-resource-areas-project.pdf.

2. $\quad$ Roberts, A., et al., Current tidal power technologies and their suitability for small-scale applications in shallow near-shore areas. Journal of Ocean Engineering and Marine Energy, 2016.

3. Denny, E., The economics of tidal energy. Energy Policy, 2009. 37(5): p. 1914-1924.

4. MeyGen Limited. The Project. 2013; Available from: http://www.meygen.com/the-project/.

5. Afshar, M. and M. Rohani, Water hammer simulation by implicit method of characteristic. International Journal of Pressure vessels and piping, 2008. 85(12): p. 851-859.

6. Bonin, C., Water-hammer damage to Oigawa power station. Journal of Engineering for Power, 1960. 82(2): p. 111-116.

7. Young, B., Generic design of ram pumps. Proceedings of the Institution of Mechanical Engineers, Part A: Journal of Power and Energy, 1998. 212(2): p. 117-124.

8. Chaudhry, M., Applied hydraulic transients. 1979, Springer.

9. Smith, P., BASIC hydraulics. 1982: Butterworth Scientific.

10. MathWorks, MATLAB and Image Processing Toolbox Release 2014a. 2014: Natick, Massachusetts, United States.

11. Aslani, N., et al., Simulation of gait asymmetry and energy transfer efficiency between unilateral and bilateral amputees. Sports Engineering, 2016: p. 1-8.

12. Chartrand, R., Numerical differentiation of noisy, nonsmooth data. ISRN Applied Mathematics, 2011. 2011.

13. MathWorks. Peak analysis. 2016 [cited 201627 May]; Available from: $\quad$ http://uk.mathworks.com/help/signal/examples/peakanalysis.html.

14. Evans, D. and R. Porter, Hydrodynamic characteristics of an oscillating water column device. Applied Ocean Research, 1995. 17(3): p. 155-164.

15. Vantorre, M., R. Banasiak, and R. Verhoeven, Modelling of hydraulic performance and wave energy extraction by a point absorber in heave. Applied Ocean Research, 2004. 26(1): p. 61-72. 